\title{
Ultrasound detection of diaphragm position in the region for lung monitoring by electrical impedance tomography during laparoscopy
}

\author{
Kristyna Buzkovaa , Martin Muller ${ }^{\mathrm{a}, \mathrm{b}}$, Ales Rara ${ }^{\mathrm{a}, \mathrm{b}}$, Karel Roubik ${ }^{\mathrm{a}}$, Tomas Tyll ${ }^{\mathrm{b}}$
}

\begin{abstract}
Background and Aims. During laparoscopic surgery, a capnoperitoneum is created to optimize the operating space for surgeons. One effect of this is abdominal pressure which alters the physiological thoraco-abdominal configuration and pushes the diaphragm and lungs cranially. Since the lung image acquired by electrical impedance tomography (EIT) depends on the conditions within the thorax and abdomen, it is crucial to know the diaphragm position to determine the effect of diaphragm shift on EIT thorax image.

Methods. The presence of diaphragm in the region of EIT measurement was determined by ultrasound in 20 patients undergoing laparoscopic surgery. Data were obtained in the supine position during spontaneous breathing in a mechanically ventilated patient under general anesthesia with muscle relaxation and in a mechanically ventilated patient under general anesthesia with muscle relaxation during capnoperitoneum.

Results. The diaphragm was shifted cranially during capnoperitoneum. The diaphragm detection rate rose by $10 \%$ during capnoperitoneum at the fifth intercostal space, from $55 \%$ to $65 \%$ and by $10 \%$ from $0 \%$ at mid-sternal level compared to mechanical ventilation without capnoperitoneum.

Conclusion. The diaphragm was detected in the area contributing to the creation of the thoracic EIT image. Considering the cranial shift of diaphragm caused by excessive intra-abdominal pressure, the impedance changes in the abdomen and the principle of EIT, we assume there could be a significant impact on EIT image of the thorax acquired during capnoperitoneum. For this reason, for lung monitoring using EIT during capnoperitoneum, the manufacturer's recommendation for electrode belt position is not appropriate.
\end{abstract}

The study was registered in ClinicalTrials.gov with an identifier NCT03038061.

Key words: capnoperitoneum, electrical impedance tomography, diaphragm, electrode belt position

Received: May 26, 2017; Accepted with revision: February 7, 2018; Available online: February 21, 2018

https://doi.org/10.5507/bp.2018.005

${ }^{a}$ Department of Biomedical Technology, Faculty of Biomedical Engineering, Czech Technical Universty in Prague, Czech Republic ${ }^{b}$ Department of Anesthesiology, Resuscitation and Intensive Care Medicine First Faculty of Medicine Charles University and the Military University Hospital Prague, Czech Republic

Corresponding author: Kristyna Buzkova, e-mail: kristyna.buzkova@fbmi.cvut.cz

\section{INTRODUCTION}

Electrical impedance tomography (EIT) is a noninvasive, radiation free bedside alternative, in some applications, to conventional imaging systems such as X-ray or CT. It can provide information about the distribution of tissue impedance within a selected body cross-section. When monitoring ventilation using the EIT system PulmoVista 500 (Dräger Medical GmbH, Lübeck, Germany), it is recommended by the manufacturer to place the electrode belt between the $4^{\text {th }}$ and $6^{\text {th }}$ intercostal space determined at the parasternal line. From the literature $^{1}$, the upper limits of the diaphragm are at the level of the fourth intercostal space. In order to avoid artifacts caused by diaphragmatic movement, the manufacturer does not recommend placing the electrode belt lower than the $5^{\text {th }}$ intercostal space for the patients with a likely elevated position of the diaphragm ${ }^{2}$. However, there are many studies pointing out missing data about optimal EIT belt position ${ }^{3}$. As Jan Karsten et $\mathrm{al}^{3}$. and many others ${ }^{4-6}$ have described, placement of the electrode belt at juxtadiaphragmatic levels can provide inaccurate information about the distribution of ventilation.
During laparoscopic surgery, capnoperitoneum is created in order to optimize the working space for surgeons. To ensure suitable surgical conditions, there is increased pressure to $12-14 \mathrm{mmHg}^{7.9}$ of $\mathrm{CO}_{2}$ in the abdomen for the duration of surgery. As a side effect, the abdominal pressure changes the physiological thoraco-abdominal configuration and most importantly pushes the diaphragm and the lungs cranially, as evidenced by both preclinical ${ }^{11}$ and clinical ${ }^{12,13}$ studies. The forced position of the diaphragm and the lungs has not been clearly analyzed in human patients and raises the question whether a proper knowledge of thoraco-abdominal configuration during laparoscopic surgery is necessary for lung monitoring methods, such as EIT. Until today, there is no study on the lung or diaphragm position changes more specifically during laparoscopic surgery. Since the EIT image depends on the conditions within the thorax and abdomen, it is crucial to know the diaphragm position to understand the effect of a cranial shift of the diaphragm on EIT images of the thorax.

The aim of this study is to examine the change in position of the diaphragm when capnoperitoneum is induced and to determine whether the diaphragm is present di- 
rectly in the region monitored by electrical impedance tomography at the conventional position of an electrode belt recommended by the manufacturer.

\section{METHODS}

A prospective interventional study was approved by the ethics committee of the Military University Hospital in Prague on November 10, 2015. The study was registered in ClinicalTrials.gov with an identifier NCT03038061. The study sample consisted of 20 patients (10 males, 10 females), average age $55.75 \pm 15.02$ years, BMI $25.76 \pm$ $3.92 \mathrm{~kg} / \mathrm{m}^{2}$. All patients enrolled in the study underwent laparoscopic surgery with capnoperitoneum (types of surgeries are specified in Table 1). The exclusion criteria were extreme obesity $\left(\mathrm{BMI}>40 \mathrm{~kg} / \mathrm{m}^{2}\right)$ and standard exclusion criteria for EIT measurement defined by the EIT device manufacturer ${ }^{2}$.

The position of the diaphragm was determined in the supine position in three phases: (1) during spontaneous breathing, (2) during mechanical ventilation under general anesthesia with muscle relaxation and (3) during mechanical ventilation under general anesthesia with muscle relaxation and capnoperitoneum. The tilt of the final position of the patients depended on the diagnosis; Trendelenburg position (up to $30^{\circ}$ tilt) was used in 11 patients, reverse Trendelenburg position (up to $40^{\circ}$ tilt) was used in 9 patients. General anesthesia was managed as combined supplemented (induction was performed with propofol, sevofluran/isofluran and bolus administration of sufentanil). During surgery, myorelaxation was ensured by non-depolarizing muscle relaxants (rocuronium, or cisatracurium). Open airways were maintained by orotracheal intubation, volume controlled ventilation (VCV) mode of mechanical ventilation was used with tidal volumes of 6-8 $\mathrm{mL} / \mathrm{kg}$, positive end-expiration pressure (PEEP) $5 \mathrm{cmH}_{2} \mathrm{O}$, respiratory rate was managed according to capnography (end-tidal concentration, $\mathrm{EtCO}_{2}$ ) to sustain normocapnia (4-5 $\mathrm{kPa} \mathrm{pCO}$ ), fraction of inspired oxygen $\left(\mathrm{FiO}_{2}\right)$ was $50 \%$. High flow insufflator units Olympus UHI-3 and UHI-4 (Olympus Surgical Technologies, Hamburg, Germany) were used for introduction and maintenance of capnoperitoneum. Pressure of the insufflation gas was set to $12-15 \mathrm{mmHg}$.

The presence of the diaphragm or the liver was determined using ultrasound Ultrasonix Sonix Touch
(Ultrasonix Medical Corporation, Richmond, Canada) during whole breathing cycles, regardless whether the tissues occurred during inspiration, expiration or both. The detection was performed in an anterior axillary line at two defined levels: at the level of the fifth intercostal space and in the middle of the sternum. A convex ultrasound probe was used. The probe was placed perpendicular to the chest wall, parallel to the ribs and in the transverse plane.

To determine whether the diaphragm or the liver was present in the region of EIT measurement, the distance between the upper edge of the sternum and the fifth intercostal space $\left(D_{5}\right)$ and the distance between the upper edge of sternum and diaphragm $\left(\mathrm{D}_{\mathrm{d}}\right)$ were measured. The area involved in EIT image formation, as declared by the manufacturer of EIT system PulmoVista 500, is $\pm 10 \mathrm{~cm}$ from the plane of the electrode belt ${ }^{2}$. For the cases when $\mid \mathrm{Dd}$ - D5 $\mid<10 \mathrm{~cm}$ applied, the presence of the diaphragm or liver was considered as confirmed. The two-tailed pair t-test was used for comparing the measured distances of diaphragm from the upper edge of sternum (spontaneous breathing vs. during capnoperitoneum, artificial ventilation vs. during capnoperitoneum). $P$-value of 0.05 was statistically significant.

\section{RESULTS}

Table 2 shows the number of positive detections of the diaphragm at the fifth intercostal space and at the mid-sternal level for spontaneously breathing patients, for mechanically ventilated patients under general anesthesia with muscle relaxation and for mechanically ventilated patients under general anesthesia with muscle relaxation and capnoperitoneum for the whole group and for each of the positions. The detection rate rose by $10 \%$ during capnoperitoneum at the fifth intercostal space, from $55.0 \%$ for ventilated patients to $65.0 \%$ for ventilated patients with capnoperitoneum and by $10 \%$ from $0 \%$ to $10 \%$ at the midsternal level compared to spontaneously breathing and mechanically ventilated patients. The presence of the diaphragm in the region of EIT lung monitoring for spontaneously breathing patients, for mechanically ventilated patients under general anesthesia with muscle relaxation and for mechanically ventilated patients under general anesthesia with muscle relaxation and capnoperitoneum is shown in Table 3. It is evident that in all cases except

Table 1. Types, patient position and numbers of performed surgeries.

\begin{tabular}{llc}
\hline Type of surgery & Position & Number of patients \\
\hline Laparoscopic cholecystectomy & Reverse Trendelenburg & 8 \\
Laparoscopic hernioplasty and fundoplication & & 1 \\
Robotic prostatectomy & Trendelenburg & 5 \\
Laparoscopic assisted vaginal hysterectomy & & 2 \\
Laparoscopic endometriosis elimination & & 2 \\
Laparoscopy for extra-uterine gravidity & & 1 \\
Laparoscopic rectal resection & & 1 \\
\hline
\end{tabular}


Table 2. The positive diaphragm detection at the fifth intercostal space and at the mid-sternal level for spontaneously breathing patients (Spontaneous), for mechanically ventilated patients under general anesthesia with muscle relaxation (Ventilated) and for mechanically ventilated patients under general anesthesia with muscle relaxation and capnoperitoneum (Ventilated $+\mathrm{CP}$ ) divided into patients undergoing surgery in the Trendelenburg position (CP, Trendelenburg) and reverse Trendelenburg position (CP, reverse Trendelenburg).

\begin{tabular}{llccccc}
\hline & & Spontaneous & Ventilated & $\begin{array}{c}\text { Ventilated } \\
+ \text { CP }\end{array}$ & $\begin{array}{c}\text { CP, } \\
\text { Trendelenburg }\end{array}$ & $\begin{array}{c}\text { CP, reverse } \\
\text { Trendelenburg }\end{array}$ \\
\hline No. of patients & & 20 & 20 & 20 & 11 & 9 \\
Fifth intercostal space & No. of detections & 12 & 11 & 13 & 8 & 5 \\
\multirow{4}{*}{ Mid-sternal level } & Detection rate (\%) & 60.0 & 55.0 & 65.0 & 72.7 & 55.5 \\
& No. of detections & 0 & 0 & 2 & 2 & 0 \\
& Detection rate (\%) & 0.0 & 0.0 & 10.0 & 18.2 & 0.0 \\
\hline
\end{tabular}

Table 3. The presence of the diaphragm in the region of EIT measurement $\left( \pm 10 \mathrm{~cm}\right.$ from the electrode belt plane at $5^{\text {th }}$ intercostal space) for spontaneously breathing patients (Spontaneous), for mechanically ventilated patients under general anesthesia with muscle relaxation (Ventilated) and for mechanically ventilated patients under general anesthesia with muscle relaxation and capnoperitoneum (Ventilated $+\mathrm{CP}$ ) divided into patients undergoing surgery in the Trendelenburg position (CP, Trendelenburg) and the reverse Trendelenburg position (CP, reverse Trendelenburg).

\begin{tabular}{|c|c|c|c|c|c|}
\hline & Spontaneous & Ventilated & Ventilated $+\mathrm{CP}$ & $\begin{array}{c}\text { CP, } \\
\text { Trendelenburg }\end{array}$ & $\begin{array}{l}\text { CP, reverse } \\
\text { Trendelenburg }\end{array}$ \\
\hline No. of patients & 20 & 20 & 20 & 11 & 9 \\
\hline No. of detections & 20 & 20 & 19 & 10 & 9 \\
\hline Detection rate $(\%)$ & 100.0 & 100.0 & 95.0 & 91.0 & 100.0 \\
\hline $\begin{array}{l}\text { Avg. distance between diaphragm } \\
\text { and } 5^{\text {th }} \text { intercostal space }(\mathrm{cm})\end{array}$ & -0.1 & 0.3 & -1.6 & -2.7 & -0.1 \\
\hline $\begin{array}{l}\text { STD - Distance between diaphragm } \\
\text { and } 5^{\text {th }} \text { intercostal space }(\mathrm{cm})\end{array}$ & 2.2 & 2.6 & 4.3 & 4.8 & 3.4 \\
\hline
\end{tabular}

one during capnoperitoneum, (100\%, 100\% and 95\%) the diaphragm was detected in the region of standard EIT measurement (i.e. $4-6^{\text {th }}$ intercostal space). The average distance between the diaphragm and the 5 th intercostal space rose from $-0.1 \mathrm{~cm}$ in spontaneously breathing patients to $-1.7 \mathrm{~cm}$ in mechanically ventilated patients with capnoperitoneum. The furthest distance $(-2.7 \mathrm{~cm})$ was detected in the Trendelenburg position with capnoperitoneum. In some cases, the diaphragm was shifted cranially beyond the area examined by ultrasound, and the liver was detected instead. $P$-values for two-tailed paired t-test were 0.002 (spontaneous breathing vs. during capnoperitoneum) and 0.005 (artificial ventilation vs. during capnoperitoneum). Both values are statistically significant.

\section{DISCUSSION}

The main finding of the study was that the diaphragm is almost always present at the area contributing to the EIT image of the thorax for all analyzed cases - for spontaneously breathing patients (100\%), mechanically ventilated patients under general anesthesia with muscle relaxation $(100 \%)$ and for mechanically ventilated patients under general anesthesia with muscle relaxation and capnoperitoneum (95\%). Another important finding is that the diaphragm is cranially shifted during capnoperitoneum. In $10 \%$ of cases during capnoperitoneum, the diaphragm was detected even at the mid-sternal level. It is also evident (as supported by the statistical significance) that the diaphragm is shifted more cranially in mechanically ventilated patients with capnoperitoneum (as shown by the negative distance in Table 3 ), whereas mechanical ventilation shifts the diaphragm caudally (as shown by the positive distance in Table 3 ).

However, several limitations of this study should be noted. Each patient is anatomically different, so the level of the fifth intercostal space does not always correspond with the exact same level of the lungs. The accuracy of the ultrasound method for detection of the diaphragm or the liver is considered to be sufficient; there might have been only some inaccuracies in the measured values of the distance between the upper edge of the sternum and the fifth intercostal space and the distance between the upper edge of sternum and the diaphragm, which should not have a significant impact. It is also difficult to maintain the exact position of an ultrasound probe in the patient covered by surgical drapes. Therefore, the detected position of the diaphragm cannot be considered as completely accurate but, based on the resolution of EIT, the deviation is acceptable. Also, the diaphragm is not always evident sonographically; therefore, the detection of the liver was also considered as a positive result proving the cranial shift of the diaphragm.

It is expected that capnoperitoneum will have an effect on the EIT image of the thorax, if the standard methodology for EIT monitoring is followed. The thoraco-abdominal configuration significantly contributes to the final EIT image. Therefore, the following issue is raised: It is not clear how the EIT image would be affected, when gas 
occurs in the peritoneum. Since gas is completely nonconducting, it could cause significant changes in EIT image. Although it was proven that the diaphragm reaches into the region monitored by EIT with no capnoperitoneum induced, it could cause much greater effect when capnoperitoneum is present, because the impedance of the abdomen changes crucially. Another study should be performed on the problem of EIT monitoring during capnoperitoneum.

Also, it is evident that during capnoperitoneum, the electrode belt should not be placed in the standard position as recommended by the manufacturer. Our results show that the EIT signal will not be useable for monitoring lungs with the electrode belt in the standard position. Another study should be performed on EIT monitoring during capnoperitoneum, analyzing the EIT signal for the cases when the electrode belt is placed more cranially.

\section{CONCLUSION}

The study has shown that during laparoscopic surgery when capnoperitoneum is induced, in the level of the fifth intercostal space, which is a conventional position of the electrode belt for EIT lung monitoring, the diaphragm or the liver is present at $65 \%$ of the patients. In $95 \%$ cases, the diaphragm was detected at the area contributing to the creation of the EIT image of the thorax $( \pm 10 \mathrm{~cm}$ from the electrode belt plane). Considering the possible cranial shift of the diaphragm caused by excessive intra-abdominal pressure, the impedance changes in the abdomen and the principle of EIT, we predict there could be a significant adverse impact on EIT image of the thorax when acquired during capnoperitoneum. For lung monitoring using EIT during capnoperitoneum, the manufacturer's recommendation of electrode belt position is unsuitable.

Acknowledgement: The study was supported by the grant SGS16/258/OHK4/3T/17.

Author contributions: KR, TT: conceived the idea and supervised the study; MM, AR: data collection, KB, KR, MM: study design, data interpretation, manuscript writing. All authors read and approved the final manuscript. Conflict of interest statement: The authors state that there are no conflicts of interest regarding the publication of this article.

\section{REFERENCES}

1. Scott-Conner CE, Dawson DL. Operative Anatomy, 3rd ed. Lippincott \&Wilkins, Philadelphia; 2009.

2. Teschner E, Imhoff M. Electrical impedance tomography: The realization of regional lung monitoring. Dräger Medical GmbH EIT Booklet, Germany, 2011.

3. Karsten J, Steuber T, Voigt N, Teschner E, Heinze H. Influence of different electrode belt positions on electrical impedance tomography imaging of regional ventilation: a prospective observational study. Crit Care 2016;20:3.

4. Bikker IG, Preis C, Egal M, Bakker J, Gommers D. Electrical impedance tomography measured at two thoracic levels can visualize the ventilation distribution changes at the bedside during a decremental positive end-expiratory lung pressure trial. Crit Care 2011;15:R193.

5. Newell JC, Isaacson D, Cheney M, Saulnier GJ, Gisser DG, Boble JC, Cook RD, Edic PM, Newton CA. In Vivo impedance images using sinusoidal current patterns. University College London, 1993, Clinical and Physiological Applications of Electrical Impedance Tomography, Chapter Five.

6. Reifferscheid F, Elke G, Pulletz S, Gawelczyk B, Lautenschläger I, Steinfath M, Weiler N, Frerichs I. Regional ventilation distribution determined by electrical impedance tomography: reproducibility and effects of posture and chest plane. Respirology 2011;16(3):52331.

7. Valenza F, Chevallard G, Fossali T, Salice V, Pizzocri M, Gattinoni L. Management of mechanical ventilation during laparoscopic surgery. Best Pract Res Clin Anaesthesiol 2010;24(2):227-41.

8. Staehr-Rye AK, Rasmussen LS, Rosenberg J, Steen-Hansen C, Nielsen TF, Rosenstock CV, Clausen HV, Sørensen MK, von H. Regeur J, Gätke MR. Minimal impairment in pulmonary function following laparoscopic surgery. Acta Anaesthesiol Scand 2014;58:198-205.

9. Andersson $L E$, Bååth $M$, Thörne $A$, Aspelin $P$, Odeberg-Wernerman S. Effect of carbon dioxide pneumoperitoneum on development of atelectasis during anesthesia, examined by spiral computed tomography. Anesthesiology 2005;102(2):293-9.

10. Joris J, Kaba A, Lamy M. Postoperative spirometry after laparoscopy for lower abdominal or upper abdominal surgical procedures. $\mathrm{Br} J$ Anesth 1997;79(4):422-6.

11. Vlot J, Wijnen R, Stolker RJ, Bax K. Optimizing working space in porcine laparoscopy: CT measurement of the effects of intra-abdominal pressure. Surg Endosc 2013;27(5):1668-73.

12. Karsten J, Luepschen $H$, Grossherr M, Bruch HP, Leonhardt S, Gehring $\mathrm{H}$, Meier T. Effect of PEEP on regional ventilation during laparoscopic surgery monitored by electrical impedance tomography. Acta Anaesthesiol Scand 2011;55(7):878-86.

13. Andersson LE, Bååth $M$, Thörne $A$, Aspelin $P$, Odeberg-Wernerman S. Effect of carbon dioxide pneumoperitoneum on development of atelectasis during anesthesia, examined by spiral computed tomography. Anesthesiology 2005;102(2):293-9. 